\title{
Fluctuation theorem for entropy production during effusion of a relativistic ideal gas
}

\author{
B. Cleuren, ${ }^{1}$ K. Willaert, ${ }^{1}$ A. Engel, ${ }^{2}$ and C. Van den Broeck ${ }^{1}$ \\ ${ }^{1}$ Hasselt University, B-3590 Diepenbeek, Belgium \\ ${ }^{2}$ Universität Oldenburg, Institut für Physik, 26111 Oldenburg, Germany
}

(Received 24 September 2007; revised manuscript received 15 November 2007; published 14 February 2008)

\begin{abstract}
The probability distribution of the entropy production for the effusion of a relativistic ideal gas is calculated explicitly. This result is then extended to include particle and antiparticle pair production and annihilation. In both cases, the fluctuation theorem is verified.
\end{abstract}

DOI: 10.1103/PhysRevE.77.022103

PACS number(s): 05.70.Ln, 05.40.-a, 05.20.-y

\section{INTRODUCTION}

The fluctuation theorem [1] can be seen as a natural extension to far from equilibrium situations of the pioneering work of Onsager on the reciprocity relations. The theorem has been proven in a variety of settings, including thermostated systems [2], Markov processes [3], classical Hamiltonian dynamics $[4,5]$, quantum dynamics, quantum field theory [6], and relativity [7]. The wide range of validity of the theorem reflects the fact that it derives from two basic ingredients of the fundamental laws of nature: time reversibility and time translational invariance. The effusion of gases provides a simple setting in which the fluctuation theorem can be verified on the basis of freshmen's physics $[8,9]$. The effusion of radiation (photons) can be discussed on a similar basis [10]. In this paper, we show that the fluctuation theorem is also valid for the effusion of classical relativistic particles, including particle and antiparticle pair production and annihilation.

\section{FLUCTUATION THEOREM FOR EFFUSION OF A RELATIVISTIC IDEAL GAS}

Two relativistic ideal gases $A$ and $B$ are initially in equilibrium in containers at given densities $\rho_{i}$ and temperature $T_{i}$, $i \in\{A, B\}$ while separated by a common adiabatic wall [11]. At time $t=0$, a small hole with surface area $\sigma$ is opened, allowing an exchange of particles and energy between the two gases during a (fixed) time interval of length $t$. We assume the presence of an energy filter, so that only particles with total energy in the range $\left[E_{1}, E_{2}\right]$ can cross. The dimensions of the hole are supposed to be small compared to the mean free path of the gas particles, and the reservoirs sufficiently large so that the equilibrium state in both parts is not affected by the exchange. In particular, the temperatures and densities in both reservoirs remain constant. Hence, the change in entropy in the total system, upon a total transfer of energy $\Delta U$ and of particles $\Delta N$ from $A$ to $B$ during $t$, is given by

$$
\begin{aligned}
\Delta S & =-\frac{1}{T_{A}} \Delta U+\frac{\mu_{A}}{T_{A}} \Delta N+\frac{1}{T_{B}} \Delta U-\frac{\mu_{B}}{T_{B}} \Delta N \\
& =\mathcal{A}_{U} \Delta U+\mathcal{A}_{N} \Delta N .
\end{aligned}
$$

We introduced, in accordance with the definitions from irreversible thermodynamics, the following thermodynamic forces (affinities) for energy and particle flow:

$$
\begin{gathered}
\mathcal{A}_{U}=\frac{1}{T_{B}}-\frac{1}{T_{A}}, \\
\mathcal{A}_{N}=\frac{\mu_{A}}{T_{A}}-\frac{\mu_{B}}{T_{B}}=k \ln \left[\frac{\rho_{A} T_{B} K_{2}\left(m c^{2} / k T_{B}\right)}{\rho_{B} T_{A} K_{2}\left(m c^{2} / k T_{A}\right)}\right],
\end{gathered}
$$

with $K_{2}$ a modified Bessel function of the second kind. The expression for the chemical potential reads

$$
\mu=-k T \ln \left(\frac{4 \pi(m c)^{3}}{\rho h^{3}} \frac{K_{2}\left(m c^{2} / k T\right)}{m c^{2} / k T}\right) .
$$

We use the convention that the internal energy $U$ of the gas comprises both the kinetic energy and the rest energy of the particles $\left(m c^{2}\right)$. Note that the thermodynamic force $\mathcal{A}_{N}$ diverges when one of the (infinitely large) reservoirs is empty, so that free effusion into unlimited space, implying an infinitely large entropy production, corresponds to a singular limit.

We will show below that the joint probability density $P_{t}(\Delta U, \Delta N)$ satisfies the following detailed fluctuation theorem:

$$
\frac{P_{t}(\Delta U, \Delta N)}{P_{t}(-\Delta U,-\Delta N)}=e^{\Delta S / k}
$$

with $\Delta S$ given by Eq. (1). This encompasses as a special case the more familiar form of the fluctuation theorem $P_{t}(\Delta S) / P_{t}(-\Delta S)=\exp \{\Delta S / k\}$. Since the increments of $\Delta U$ and $\Delta N$ in successive time intervals are independent, one has that

$$
\left\langle e^{-\left(\lambda_{U} \Delta U+\lambda_{N} \Delta N\right)}\right\rangle=e^{-t Q\left(\lambda_{U}, \lambda_{N}\right)},
$$

where $Q\left(\lambda_{U}, \lambda_{N}\right)$ is the cumulant generating function. In terms of the latter, the detailed fluctuation theorem reads

$$
Q\left(\lambda_{U}, \lambda_{N}\right)=Q\left(\mathcal{A}_{U} / k-\lambda_{U}, \mathcal{A}_{N} / k-\lambda_{N}\right) .
$$

\section{MASTER EQUATION AND CUMULANT GENERATING FUNCTION}

During a small time interval $d t$, the contributions to the 
quantities $\Delta U$ and $\Delta N$ result from individual particle transport across the hole. The corresponding transition rate $T_{i \rightarrow j}(E)$ to observe a particle with energy $E$ crossing the hole from $i \rightarrow j$ is given by

$$
T_{i \rightarrow j}(E)=\frac{\sigma \rho_{i}}{4 c^{2}(m c)^{3}} \frac{\left(m c^{2} / k T_{i}\right) e^{-E / k T_{i}}}{K_{2}\left(m c^{2} / k T_{i}\right)}\left(E^{2}-m^{2} c^{4}\right) .
$$

The derivation of this result is similar to the nonrelativistic case [8], but with the velocities described by the Jüttner distribution [12]. The probability density $P_{t}(\Delta U, \Delta N)$ then obeys the following master equation:

$$
\begin{aligned}
\frac{\partial}{\partial t} P_{t}(\Delta U, \Delta N)= & \int_{E_{1}}^{E_{2}} T_{A \rightarrow B}(E) P_{t}(\Delta U-E, \Delta N-1) d E \\
& +\int_{E_{1}}^{E_{2}} T_{B \rightarrow A}(E) P_{t}(\Delta U+E, \Delta N+1) d E \\
& -P_{t}(\Delta U, \Delta N) \int_{E_{1}}^{E_{2}}\left[T_{A \rightarrow B}(E)+T_{B \rightarrow A}(E)\right] d E,
\end{aligned}
$$

subject to the initial condition $P_{0}(\Delta U, \Delta N)=\delta(\Delta U) \delta_{\Delta N, 0}$. Multiplying the master equation with $\exp \left\{-\lambda_{U} \Delta U-\lambda_{N} \Delta N\right\}$ and subsequent integration over $\Delta U$ and summation over $\Delta N$ leads to the following analytical result:

$$
Q\left(\lambda_{U}, \lambda_{N}\right)=I_{A}(0,0)-I_{A}\left(\lambda_{U}, \lambda_{N}\right)+I_{B}(0,0)-I_{B}\left(-\lambda_{U},-\lambda_{N}\right),
$$

where we define

$$
\begin{aligned}
& I_{i}\left(\lambda_{U}, \lambda_{N}\right)=\int_{E_{1}}^{E_{2}} T_{i \rightarrow j}(E) e^{-\lambda_{U} E-\lambda_{N}} d E \\
& =\frac{e^{-\lambda_{N}} \sigma \rho_{i}}{4 m^{2} c^{3}} \frac{\left(k T_{i}\right)^{2}\left(1+\lambda_{U} k T_{i}\right)^{-3}}{K_{2}\left(m c^{2} / k T_{i}\right)} \\
& \times\left[e ^ { - ( 1 / k T _ { i } + \lambda _ { U } ) E _ { 1 } } \left\{2+2 E_{1}\left(k T_{i}\right)^{-1}+\lambda_{U}\right.\right. \\
& \left.+\left(E_{1}^{2}-m^{2} c^{4}\right)\left(\left(k T_{i}\right)^{-1}+\lambda_{U}\right)^{2}\right\} \\
& -e^{-\left(1 / k T_{i}+\lambda_{U}\right) E_{2}\left\{2+2 E_{2}\left(k T_{i}\right)^{-1}+\lambda_{U}\right.} \\
& \left.\left.+\left(E_{2}^{2}-m^{2} c^{4}\right)\left(\left(k T_{i}\right)^{-1}+\lambda_{U}\right)^{2}\right\}\right] \text {. }
\end{aligned}
$$

Equation (9), together with Eq. (10), is the central result of this paper. A tedious calculation, using the explicit expressions for the thermodynamic forces given in Eq. (2), reveals that this expression indeed verifies the detailed fluctuation theorem Eq. (6).

\section{CUMULANTS}

A Taylor expansion of the cumulant generating function gives immediately the expressions of the joint cumulants $\kappa_{i j}$ (of power $i$ in the energy flux and power $j$ in the particle flux):

$$
Q\left(\lambda_{U}, \lambda_{N}\right)=-\frac{1}{t} \sum_{i, j} \frac{(-1)^{i+j} \lambda_{U}^{i} \lambda_{N}^{j}}{i ! j !} \kappa_{i j}
$$

As the higher order expressions quickly become unwieldy, we will only present the first order results, namely, $\kappa_{10}$ $=\langle\Delta U\rangle$ and $\kappa_{01}=\langle\Delta N\rangle$. These results will be used in the next section to validate the Onsager symmetry:

$$
\begin{aligned}
& \langle\Delta U\rangle=\frac{t \sigma \rho_{A}}{4 m^{2} c^{3} K_{2}\left(m c^{2} / k T_{A}\right)}\left\{e^{-E_{1} / k T_{A}}\left[E_{1}^{3}-E_{1} m^{2} c^{4}+3 E_{1}^{2} k T_{A}-m^{2} c^{4} k T_{A}+6 E_{1}\left(k T_{A}\right)^{2}+6\left(k T_{A}\right)^{3}\right]\right. \\
& \left.-e^{-E_{2} / k T_{A}}\left[E_{2}^{3}-E_{2} m^{2} c^{4}+3 E_{2}^{2} k T_{A}-m^{2} c^{4} k T_{A}+6 E_{2}\left(k T_{A}\right)^{2}+6\left(k T_{A}\right)^{3}\right]\right\}-\frac{t \sigma \rho_{B}}{4 m^{2} c^{3} K_{2}\left(m c^{2} / k T_{B}\right)}\left\{e^{-E_{1} / k T_{B}\left[6\left(k T_{B}\right)^{3}-E_{1} m^{2} c^{4}\right.}\right. \\
& \left.\left.+3 E_{1}^{2} k T_{B}-m^{2} c^{4} k T_{B}+6 E_{1}\left(k T_{B}\right)^{2}+E_{1}^{3}\right]-e^{-E_{2} / k T_{B}}\left[6\left(k T_{B}\right)^{3}-E_{2} m^{2} c^{4}+3 E_{2}^{2} k T_{B}-m^{2} c^{4} k T_{B}+6 E_{2}\left(k T_{B}\right)^{2}+E_{2}^{3}\right]\right\}, \\
& \langle\Delta N\rangle=\frac{t \sigma \rho_{A}}{4 m^{2} c^{3} K_{2}\left(m c^{2} / k T_{A}\right)}\left\{e^{-E_{1} / k T_{A}}\left[E_{1}^{2}-m^{2} c^{4}+2 E_{1} k T_{A}+2\left(k T_{A}\right)^{2}\right]-e^{-E_{2} / k T_{A}}\left[E_{2}^{2}-m^{2} c^{4}+2 E_{2} k T_{A}+2\left(k T_{A}\right)^{2}\right]\right\}
\end{aligned}
$$

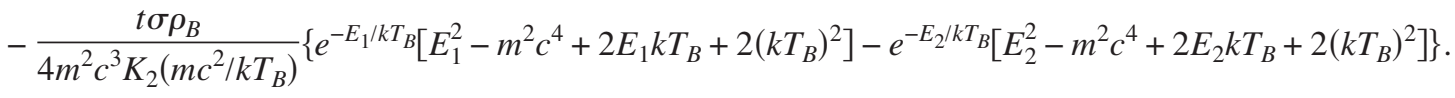

Both $\langle\Delta U\rangle$ and $\langle\Delta N\rangle$ grow linear in time, in accordance with the fact that their increments are independent. 


\section{ONSAGER RELATION}

The average entropy production during the effusion process is obtained by averaging Eq. (1) and taking the time derivative

$$
\frac{d}{d t}\langle\Delta S\rangle=\mathcal{A}_{U} \mathcal{J}_{U}+\mathcal{A}_{N} \mathcal{J}_{N}
$$

where we introduce the macroscopic fluxes $\mathcal{J}_{U}=\frac{d}{d t}\langle\Delta U\rangle$ and $\mathcal{J}_{N}=\frac{d}{d t}\langle\Delta N\rangle$ corresponding to energy and particle transport. These fluxes in general depend nonlinearly on the thermodynamic forces. Close to equilibrium, when the thermodynamic forces are small (that is, for small temperature and density difference), the expressions can be linearized, and written in compact matrix notation $\overline{\mathcal{J}}=L \overline{\mathcal{A}}$ using $\overline{\mathcal{J}}=\left(\mathcal{J}_{U}, \mathcal{J}_{N}\right)^{T}, \overline{\mathcal{A}}$ $=\left(\mathcal{A}_{U}, \mathcal{A}_{N}\right)^{T}$ and the Onsager matrix $L$. The explicit expression for the Onsager coefficients $L_{i j}$ are obtained from Eqs. (12) and (13)

$$
\begin{aligned}
L_{U U}= & \frac{\sigma \rho}{4 k m^{2} c^{3} K_{2}\left(m c^{2} / k T\right)} \\
& \times\left\{e ^ { - E _ { 1 } / k T } \left[E_{1}^{4}+4 E_{1}^{3} k T+12 E_{1}^{2}(k T)^{2}-E_{1}^{2} m^{2} c^{4}\right.\right. \\
& \left.+24 E_{1}(k T)^{3}-2 E_{1} k T m^{2} c^{4}+24(k T)^{4}-2 k^{2} T^{2} m^{2} c^{4}\right] \\
& -e^{-E_{2} / k T}\left[E_{2}^{4}+4 E_{2}^{3} k T+12 E_{2}^{2}(k T)^{2}-E_{2}^{2} m^{2} c^{4}\right. \\
+ & \left.\left.24 E_{2}(k T)^{3}-2 E_{2} k T m^{2} c^{4}+24(k T)^{4}-2 k^{2} T^{2} m^{2} c^{4}\right]\right\} \\
& \quad L_{U N}=L_{N U}=\frac{\sigma \rho}{4 k m^{2} c^{3} K_{2}\left(m c^{2} / k T\right)} \\
& \left.-E_{1} m^{2} c^{4}+6(k T)^{3}-k T m^{2} c^{4}\right] \\
-e^{-E_{2} / k T}\left[E_{2}^{3}+3 E_{2}^{2} k T+6 E_{2}(k T)^{2}\right. & \left.\left.-E_{2} m^{2} c^{4}+6(k T)^{3}-k T m^{2} c^{4}\right]\right\} ; \\
L_{N N}= & \sigma \rho \\
4 k m^{2} c^{3} K_{2}\left(\frac{m c^{2}}{k T}\right) & \left\{e^{-E_{1} / k T}\left[E_{1}^{2}+2 E_{1} k T+2(k T)^{2}-m c^{2}\right]\right.
\end{aligned}
$$

The symmetry property of the matrix is a direct consequence of the symmetry relation Eq. (6) of the cumulant generating function.

These expressions significantly simplify when considering an infinitesimal energy window $E_{1}=E_{0}-d E / 2$ and $E_{2}$ $=E_{0}+d E / 2\left(d E \ll E_{0}\right)$. In this case, there is a strong coupling between the particle and energy current: every particle that crosses carries a energy $E_{0}$ and so $\mathcal{J}_{U}=E_{0} \mathcal{J}_{N}$. This is reflected in the Onsager matrix, after expanding the $L_{i j}$ to first order in $d E$ :

$$
L_{U U}=E_{0}^{2} L_{N N}, \quad L_{U N}\left(=L_{N U}\right)=E_{0} L_{N N},
$$

$$
L_{N N}=\frac{\sigma \rho\left(E_{0}^{2}-m^{2} c^{4}\right) e^{-E_{0} / k T} d E}{4 k m^{2} c^{3} K_{2}\left(m c^{2} / k T\right)} .
$$

The determinant of the Onsager matrix is identically zero, as both rows are linear dependent. This opens up the possiblity, in principle, to use the system as a thermodynamic engine attaining Carnot or Curzon-Ahlborn efficiencies [13,14].

\section{PAIR PRODUCTION}

One important aspect of relativistic gases, which we have not considered so far, is the production and annihilation of particle-antiparticle pairs [15]. This must be included in a consistent treatment of relativistic effusion by considering the corresponding exchange of antiparticles between the two compartments.

In our framework, in which both reservoirs independently are (and remain) in equilibrium, this implies the existence of a second ideal gas in each reservoir consisting of antiparticles. Because of the production and annihilation, both particles and antiparticles will be created and destroyed. This process can be represented by a chemical reaction of the form $x+\bar{x} \rightleftharpoons 0$ ( $x$ represents a particle, $\bar{x}$ the corresponding antiparticle). Equilibrium is achieved when the two chemical potentials $(\mu$ and $\bar{\mu})$ satisfy $\mu+\bar{\mu}=0$. For a given temperature this relation determines the density $\bar{\rho}$ of the antiparticles. Using Eq. (3) gives

$$
\bar{\rho}=\left[4 \pi\left(\frac{m c}{h}\right)^{3} \frac{K_{2}\left(m c^{2} / k T\right)}{m c^{2} / k T}\right]^{2} \frac{1}{\rho} .
$$

Note that fluctuations of (anti)particles' number and energy around their equilibrium values can be neglected, as both reservoirs are assumed to be infinitely large.

The exchange of antiparticles through the opening between volumes $A$ and $B$ will contribute to the entropy change $\Delta S$. Since the chemical potential of the antiparticles is just the negative of the chemical potential of the particles we find instead of Eq. (1)

$$
\Delta S=\mathcal{A}_{U} \Delta U+\mathcal{A}_{N}(\Delta N-\Delta \bar{N}),
$$

with $\mathcal{A}_{U}$ and $\mathcal{A}_{N}$ given by Eq. (2). To account for the fluctuations in $\Delta S$ due to the exchange of antiparticles the joint probability distribution has to be augmented to $P(\Delta U, \Delta N, \Delta \bar{N})$, and its time evolution is now governed by the master equation

$$
\begin{aligned}
\frac{\partial}{\partial t} P_{t}(\Delta U, \Delta N, \Delta \bar{N})= & \int_{E_{1}}^{E_{2}}\left[T_{A \rightarrow B}(E) P_{t}(\Delta U-E, \Delta N-1, \Delta \bar{N})\right. \\
& +\bar{T}_{A \rightarrow B}(E) P_{t}(\Delta U-E, \Delta N, \Delta \bar{N}-1) \\
& +T_{B \rightarrow A}(E) P_{t}(\Delta U+E, \Delta N+1, \Delta \bar{N}) \\
& \left.+\bar{T}_{B \rightarrow A}(E) P_{t}(\Delta U+E, \Delta N, \Delta \bar{N}+1)\right] d E \\
& -P_{t}(\Delta U, \Delta N, \Delta \bar{N}) \int_{E_{1}}^{E_{2}}\left[T_{A \rightarrow B}(E)\right. \\
& \left.+\bar{T}_{A \rightarrow B}(E)+T_{B \rightarrow A}(E)+\bar{T}_{B \rightarrow A}(E)\right] d E,
\end{aligned}
$$


where the rates $T_{i \rightarrow j}(E)$ and $\bar{T}_{i \rightarrow j}(E)$ are defined by Eq. (7) with $\rho_{i}$ replaced by $\bar{\rho}_{i}$ in the latter case. The corresponding cumulant generating function is given by

$$
\left\langle e^{-\left(\lambda_{U} \Delta U+\lambda_{N} \Delta N+\lambda_{\bar{N}} \Delta \bar{N}\right)}\right\rangle=e^{-t Q\left(\lambda_{U}, \lambda_{N}, \lambda_{N}\right)} .
$$

The detailed fluctuation theorem acquires the form

$$
Q\left(\lambda_{U}, \lambda_{N}, \lambda_{\bar{N}}\right)=Q\left(\mathcal{A}_{U} / k-\lambda_{U}, \mathcal{A}_{N} / k-\lambda_{N}, \mathcal{A}_{\bar{N}} / k-\lambda_{\bar{N}}\right) .
$$

Similar to Eq. (9) we now find

$$
\begin{aligned}
Q\left(\lambda_{U}, \lambda_{N}, \lambda_{\bar{N}}\right)= & I_{A}(0,0)-I_{A}\left(\lambda_{U}, \lambda_{N}\right)+\bar{I}_{A}(0,0)-\bar{I}_{A}\left(\lambda_{U}, \lambda_{\bar{N}}\right) \\
& +I_{B}(0,0)-I_{B}\left(-\lambda_{U},-\lambda_{N}\right) \\
& +\bar{I}_{B}(0,0)-\bar{I}_{B}\left(-\lambda_{U},-\lambda_{\bar{N}}\right),
\end{aligned}
$$

with $I_{i}\left(\lambda_{U}, \lambda_{N}\right)$ given by Eq. (10). For $\bar{I}_{i}$ the density is $\bar{\rho}_{i}$. Using these expressions in Eq. (22) it is again straightforward to verify Eq. (21). The inclusion of pair production thus opens an additional channel for entropy production. However, due to the equilibrium relations between the chemical potentials of particles and antiparticles $(\mu+\bar{\mu}=0)$ and their respective concentrations [Eq. (17)] the fluctuation theorem remains valid.

\section{ACKNOWLEDGMENT}

B.C. acknowledges support from the Fonds voor Wetenschappelijk Onderzoek-Vlaanderen.
[1] D. J. Evans, E. G. D. Cohen, and G. P. Morriss, Phys. Rev. Lett. 71, 2401 (1993); G. Gallavotti and E. G. D. Cohen, ibid. 74, 2694 (1995); G. Gallavotti, ibid. 77, 4334 (1996); Work, Dissipation, and Fluctuations in Nonequilibrium Systems, edited by B. Derrida, P. Gaspard, and C. Van den Broeck, Vol. 8 of C. R. Physique (Académie des Sciences, Paris, 2007).

[2] D. J. Evans and D. J. Searles, Phys. Rev. E 50, 1645 (1994); M. Dolowschiák and Z. Kovács, ibid. 71, 025202(R) (2005); T. Gilbert, ibid. 73, 035102(R) (2006).

[3] J. Kurchan, J. Phys. A 31, 3719 (1998); J. L. Lebowitz and H. Spohn, J. Stat. Phys. 95, 333 (1999); D. Andrieux and P. Gaspard, J. Chem. Phys. 121, 6167 (2004); D. Andrieux and P. Gaspard, J. Stat. Mech.: Theory Exp. (2006), P01011; C. Maes, J. Stat. Phys. 95, 367 (1999).

[4] B. Cleuren, C. Van den Broeck, and R. Kawai, Phys. Rev. Lett. 96, 050601 (2006).

[5] C. Jarzynski, J. Stat. Phys. 98, 77 (2000).

[6] M. Esposito and S. Mukamel, Phys. Rev. E 73, 046129 (2006); M. Esposito, U. Harbola, and S. Mukamel, Phys. Rev.
B 75, 155316 (2007); U. Harbola, M. Esposito, and S. Mukamel, ibid. 76, 085408 (2007).

[7] A. Fingerle, C. R. Phys. 8, 696 (2007).

[8] B. Cleuren, C. Van den Broeck, and R. Kawai, Phys. Rev. E 74, 021117 (2006).

[9] K. Wood, C. Van den Broeck, R. Kawai, and K. Lindenberg, Phys. Rev. E 75, 061116 (2007).

[10] B. Cleuren and C. Van den Broeck, Europhys. Lett. 79, 30001 (2007).

[11] For simplicity we assume that the containers are stationary. The issue of gases in relative motion at relativistic speeds, can be analyzed along the lines of Ref. [9].

[12] D. Cubero, J. Casado-Pascual, J. Dunkel, P. Talkner, and P. Hänggi, Phys. Rev. Lett. 99, 170601 (2007).

[13] C. Van den Broeck, Phys. Rev. Lett. 95, 190602 (2005).

[14] C. Van den Broeck, Adv. Chem. Phys. 135, 189 (2007).

[15] H. E. Haber and H. A. Weldon, Phys. Rev. Lett. 46, 1497 (1981). 